\title{
All intra-regular generalized hypersubstitutions of type (2)
}

Ampika Boonmee

Department of Mathematics, Faculty of Science,

Chiang Mai University, Thailand

email: ampika.b.ku.src@gmail.com
Sorasak Leeratanavalee

Research Center in Mathematics and Applied Mathematics, Department of

Mathematics, Faculty of Science, Chiang Mai University, Thailand email: sorasak.1@cmu.ac.th

\begin{abstract}
A generalized hypersubstitution of type $\tau$ maps each operation symbol of the type to a term of the type, and can be extended to a mapping defined on the set of all terms of this type. The set of all such generalized hypersubstitutions forms a monoid. An element a of a semigroup $S$ is intra-regular if there is $b \in S$ such that $a=b a a b$. In this paper, we determine the set of all intra-regular elements of this monoid for type $\tau=(2)$.
\end{abstract}

\section{Introduction}

A solid variety is a variety in which every identity holds as a hyperidentity, that is, we substitute not only elements for the variables but also term operations for the operation symbols. The notions of hyperidentities and hypervarieties of a given type $\tau$ without nullary operations were studied by J. Aczèl [1], V. D. Belousov [2], W.D. Neumann [8] and W. Taylor [13]. The main tool used to study hyperidentities and hypervarieties is the concept of a hypersubstitution, introduced by K. Denecke et al. [5]. The concept of a generalized hypersubstitution was introduced by S. Leeratanavalee and K. Denecke [7]. The authors

2010 Mathematics Subject Classification: 20M05, 20M17

Key words and phrases: generalized hypersubstitution, regular element, sequence of term, completely regular, intra-regular element 
defined a binary operation on the set of all generalized hypersubstitutions and proved that this set together with the binary operation forms a monoid. In 2010, W. Puninagool and S. Leeratanavalee determined all regular elements of this monoid for type $\tau=(n)$, see [10]. The set of all completely regular elements of this monoid of type $\tau=(n)$ was determined by A. Boonmee and S. Leeratanavalee [3]. Furthermore, we found that every completely regular element is intra-regular. In the present paper, we show that the set of all completely regular elements and the set of all intra-regular elements of type $\tau=(2)$ are the same.

Let $n \geq 1$ be a natural number and let $X_{n}:=\left\{x_{1}, x_{2}, \ldots, x_{n}\right\}$ be an $n-$ element set which is called an $\mathrm{n}$-element alphabet and let its elements be called variables. Let $X:=\left\{x_{1}, x_{2}, \ldots\right\}$ be a countably infinite set of variables and $\left\{f_{i} \mid i \in I\right\}$ be a set of $n_{i}$-ary operation symbols, which is disjoint from $X$, indexed by the set $I$. To every $n_{i}$-ary operation symbol $f_{i}$ we assign a natural number $n_{i} \geq 1$, called the arity of $f_{i}$. The sequence $\tau=\left(n_{i}\right)_{i \in I}$ is called the type. For $\mathrm{n} \geq 1$, an $\mathrm{n}$-ary term of type $\tau$ is defined in the following inductive way:

(i) Every variable $x_{i} \in X_{n}$ is an $n$-ary term of type $\tau$.

(ii) If $t_{1}, \ldots, t_{n_{i}}$ are $n$-ary terms of type $\tau$ then $f_{i}\left(t_{1}, \ldots, t_{n_{i}}\right)$ is an $n$-ary term of type $\tau$.

The smallest set which contains $x_{1}, \ldots, x_{n}$ and is closed under any finite number of applications of (ii) is denoted by $W_{\tau}\left(X_{n}\right)$, and is called the set of all $n$ - ary terms of type $\tau$. The set $W_{\tau}(X):=\cup_{n=1}^{\infty} W_{\tau}\left(X_{n}\right)$ is called the set of all terms of type $\tau$.

A generalized hypersubstitution of type $\tau=\left(n_{i}\right)_{i \in I}$ is a mapping $\sigma:\left\{f_{i}\right\}$ $i \in I\} \rightarrow W_{\tau}(X)$ which does not necessarily preserve the arity. Let Hyp $p_{G}(\tau)$ be the set of all generalized hypersubstitutions of type $\tau$. In general, the usual composition of mappings can be used as a binary operation on mappings. But in the case of $\operatorname{Hyp}_{\mathrm{G}}(\tau)$ this can not be done immediately. To define a binary operation on this set, we define inductively the concept of a generalized superposition of terms $S^{m}: W_{\tau}(X)^{m+1} \rightarrow W_{\tau}(X)$ by the following steps:

(i) If $t=x_{j}, 1 \leq j \leq m$, then $S^{m}\left(x_{j}, t_{1}, \ldots, t_{m}\right):=t_{j}$.

(ii) If $t=x_{j}, m<j \in \mathbb{N}$, then $S^{m}\left(x_{j}, t_{1}, \ldots, t_{m}\right):=x_{j}$.

(iii) If $t=f_{i}\left(s_{1}, s_{2}, \ldots, s_{n_{i}}\right)$, then $S^{m}\left(t, t_{1}, \ldots, t_{m}\right):=f_{i}\left(S^{m}\left(s_{1}, t_{1}, \ldots, t_{m}\right), \ldots, S^{m}\left(s_{n_{i}}, t_{1}, \ldots, t_{m}\right)\right)$. 
We extend any generalized hypersubstitution $\sigma$ to a mapping $\widehat{\sigma}: W_{\tau}(X) \rightarrow$ $\mathrm{W}_{\tau}(\mathrm{X})$ inductively defined as follows:

(i) $\widehat{\sigma}[x]:=x \in X$,

(ii) $\widehat{\sigma}\left[f_{i}\left(t_{1}, t_{2}, \ldots, t_{n_{i}}\right)\right]:=S^{n_{i}}\left(\sigma\left(f_{i}\right), \widehat{\sigma}\left[t_{1}\right], \ldots, \widehat{\sigma}\left[t_{n_{i}}\right]\right)$, for any $n_{i}$-ary operation symbol $f_{i}$ assuming that $\widehat{\sigma}\left[t_{j}\right], 1 \leq j \leq n_{i}$ are already defined.

Now, we define a binary operation $\circ_{\mathrm{G}}$ on $\operatorname{Hyp}_{\mathrm{G}}(\tau)$ by $\sigma_{1} \circ_{\mathrm{G}} \sigma_{2}:=\widehat{\sigma}_{1} \circ \sigma_{2}$ where $\circ$ denotes the usual composition of mappings. Let $\sigma_{i d}$ be the hypersubstitution which maps each $n_{i}$-ary operation symbol $f_{i}$ to the term $f_{i}\left(x_{1}, x_{2}, \ldots, x_{n_{i}}\right)$. Then $\operatorname{Hyp}_{\mathrm{G}}(\tau)=\left(\mathrm{Hyp}_{\mathrm{G}}(\tau), \circ_{\mathrm{G}}, \sigma_{\mathrm{id}}\right)$ is a monoid [7].

From now on, we introduce some notations which will be used throughout this paper. For a type $\tau=(n)$ with an $n$-ary operation symbol $f$ and $t \in$ $W_{(n)}(X)$, we denote

$\sigma_{t}$ - the generalized hypersubstitution $\sigma$ of type $\tau=(n)$ which maps $f$ to the term $t$,

$\operatorname{var}(t)$ - the set of all variables occurring in the term $t$,

$\mathrm{vb}^{\mathrm{t}}(\mathrm{x})$ - the total number of $x$-variable occurring in the term $t$.

For a term $t \in W_{(n)}(X)$, the set $\operatorname{sub}(t)$ of its subterms is defined as follows $([11],[12])$ :

(i) if $t \in X$, then $\operatorname{sub}(t)=\{t\}$,

(ii) if $t=f\left(t_{1}, \ldots, t_{n}\right)$, then $\operatorname{sub}(t)=\{t\} \cup \operatorname{sub}\left(t_{1}\right) \cup \ldots \cup \operatorname{sub}\left(t_{n}\right)$.

Example 1 Let $\tau=(2)$ and $t \in W_{(2)}(X)$ where $t=f\left(t_{1}, t_{2}\right)$ with $t_{1}=$ $f\left(x_{3}, f\left(x_{1}, x_{4}\right)\right)$ and $t_{2}=f\left(f\left(x_{7}, x_{1}\right), f\left(x_{2}, x_{1}\right)\right)$. Then

$\operatorname{var}(t)=\left\{x_{1}, x_{2}, x_{3}, x_{4}, x_{7}\right\}$

$v^{\mathrm{t}}\left(\mathrm{x}_{1}\right)=3, v \mathrm{~b}^{\mathrm{t}}\left(\mathrm{x}_{2}\right)=1, v \mathrm{~b}^{\mathrm{t}}\left(\mathrm{x}_{3}\right)=1, v \mathrm{~b}^{\mathrm{t}}\left(\mathrm{x}_{4}\right)=1, v \mathrm{~b}^{\mathrm{t}}\left(\mathrm{x}_{7}\right)=1$,

$\operatorname{sub}\left(t_{1}\right)=\left\{t_{1}, f\left(x_{1}, x_{4}\right), x_{1}, x_{3}, x_{4}\right\}$

$\operatorname{sub}\left(t_{2}\right)=\left\{t_{2}, f\left(x_{7}, x_{1}\right), f\left(x_{2}, x_{1}\right), x_{1}, x_{2}, x_{7}\right\}$

$\operatorname{sub}(t)=\left\{t, t_{1}, t_{2}, f\left(x_{1}, x_{4}\right), f\left(x_{7}, x_{1}\right), f\left(x_{2}, x_{1}\right), x_{1}, x_{2}, x_{3}, x_{4}, x_{7}\right\}$.

\section{Sequence of terms}

In this section, we construct some tools used to characterize all intra-regular elements in $\mathrm{Hyp}_{\mathrm{G}}(2)$. These tools are called the sequence of a term and the depth of a term, respectively. 
Definition 1 Let $\mathrm{t} \in \mathrm{W}_{(\mathrm{n})}(\mathrm{X}) \backslash \mathrm{X}$ where $\mathrm{t}=\mathrm{f}\left(\mathrm{t}_{1} \ldots, \mathrm{t}_{\mathrm{n}}\right)$ for some $\mathrm{t}_{1}, \ldots \mathrm{t}_{\mathrm{n}} \in$ $W_{(\mathrm{n})}(\mathrm{X})$. For each $\mathrm{s} \in \operatorname{sub}(\mathrm{t}), \mathrm{s} \neq \mathrm{t}$, a set $\operatorname{seq}^{\mathrm{t}}(\mathrm{s})$ of sequences of $\mathrm{s}$ in $\mathrm{t}$ is defined by where $\pi_{i_{l}}: W_{(n)}(X) \backslash X \rightarrow W_{(n)}(X)$ by the formula $\pi_{i_{l}}\left(f\left(t_{1}, \ldots, t_{n}\right)\right)=$ $\mathrm{t}_{\mathrm{i}_{l}}$. Maps $\pi_{\mathfrak{i}_{l}}$ are defined for $\boldsymbol{i}_{l}=1,2, \ldots, n$.

Example 2 Let $\mathrm{t} \in \mathrm{W}_{(4)}(\mathrm{X})$ where $\mathrm{t}=\mathrm{f}\left(\mathrm{t}_{1}, \mathrm{t}_{2}, \mathrm{t}_{3}, \mathrm{t}_{4}\right)$ such that $\mathrm{t}_{1}=\mathrm{f}\left(\mathrm{x}_{3}, \mathrm{x}_{1}, \mathrm{~s}\right.$, $\left.x_{4}\right), t_{2}=x_{4}, t_{3}=\left(f\left(x_{7}, s, x_{1}, x_{4}\right), x_{4}, f\left(x_{8}, f\left(x_{3}, x_{1}, s, x_{4}\right), x_{2}, f\left(x_{3}, x_{1}, s, x_{4}\right)\right), s\right)$ and $\mathrm{t}_{4}=\mathrm{s}$ for some $\mathrm{s} \in \mathrm{W}_{(4)}(\mathrm{X})$. Then

$$
\begin{aligned}
& \operatorname{seq}^{t}(s)=\{(1,3),(3,1,2),(3,3,2,3),(3,3,4,3),(3,4),(4)\} \text {, } \\
& \operatorname{seq}^{t_{3}}(s)=\{(1,2),(3,2,3),(3,4,3),(4)\} \text {, } \\
& \operatorname{seq}^{\mathrm{t}}\left(\mathrm{t}_{1}\right)=\{(1),(3,3,2),(3,3,4)\} \\
& \operatorname{seq}^{\mathrm{t}}\left(x_{4}\right)=\{(1,4),(2),(3,1,3)\} \text {. }
\end{aligned}
$$

Lemma 1 ([4]) Let $\mathrm{t}, \mathrm{s} \in \mathrm{W}_{(\mathrm{n})}(\mathrm{X}) \backslash \mathrm{X}, \mathrm{x} \in \operatorname{var}(\mathrm{t})$ and $\operatorname{var}(\mathrm{s}) \cap \mathrm{X}_{\mathrm{n}}=\left\{\mathrm{x}_{z_{1}}, \ldots, \mathrm{x}_{z_{k}}\right\}$. If $\left(i_{1}, \ldots, i_{m}\right) \in \operatorname{seq}^{t}(x)$ where $i_{1}, \ldots, i_{m} \in\left\{z_{1}, \ldots, z_{k}\right\}$ then $x \in \operatorname{var}\left(\widehat{\sigma}_{s}[t]\right)=$ $\operatorname{var}\left(\sigma_{s} \circ_{G} \sigma_{t}\right)$ and there is $\left(a_{i_{1}}, \ldots, a_{i_{m}}\right) \in \operatorname{seq}^{\widehat{\sigma}_{s}[t]}(x)$ where $a_{i_{j}}$ is a sequence of natural numbers $j_{1}, \ldots, j_{h}$ such that $\left(j_{1}, \ldots, j_{h}\right) \in \operatorname{seq}^{s}\left(x_{i_{j}}\right)$ for all $j \in$ $\{1, \ldots, m\}$.

Let $t \in W_{(n)}(X) \backslash X$, and $t_{i} \in \operatorname{sub}(t)$. It can be possible that $t_{i}$ occurs in the term $t$ more than once, we denote

$t_{i}^{(j)}$ - subterm $t_{i}$ occurring in the $j^{\text {th }}$ order of $t$ (from the left).

Definition 2 Let $t \in W_{(n)}(X) \backslash X$ where $t=f\left(t_{1}, \ldots, t_{n}\right)$ for some $t_{1}, \ldots, t_{n} \in$ $W_{(n)}(X)$ and let $\pi_{i_{l}}: W_{(n)}(X) \backslash X \rightarrow W_{(n)}(X)$ by the formula $\pi_{i_{l}}(t)=\pi_{i_{l}}\left(f\left(t_{1}, \ldots\right.\right.$, $\left.\left.t_{n}\right)\right)=t_{i_{l}}$. Maps $\pi_{i_{l}}$ are defined for $i_{l}=1,2, \ldots, n$. For each $s^{(j)} \in \operatorname{sub}(t)$ for some $j \in \mathbb{N}$, we denote the sequence of $s^{(j)}$ in $t$ by $\operatorname{seq}^{t}\left(s^{(j)}\right)$ and denote the depth of $s^{(j)}$ in $t$ by $\operatorname{depth}^{t}\left(s^{(j)}\right)$. If $s^{(j)}=\pi_{i_{m}} \circ \ldots \circ \pi_{i_{1}}(t)$ for some $m \in \mathbb{N}$, then

$$
\operatorname{seq}^{\mathrm{t}}\left(\mathrm{s}^{(\mathfrak{j})}\right)=\left(\mathfrak{i}_{1}, \ldots, \mathfrak{i}_{\mathrm{m}}\right) \text { and } \operatorname{depth}^{\mathrm{t}}\left(\mathrm{s}^{(\mathfrak{j})}\right)=\mathrm{m} \text {. }
$$

Example 3 Let $\tau=(3)$ and let $\mathrm{t} \in \mathrm{W}_{(3)}(\mathrm{X}) \backslash \mathrm{X}$ where $\mathrm{t}=\mathrm{f}\left(\mathrm{t}_{1}, \mathrm{t}_{2}, \mathrm{t}_{3}\right)$ such that $t_{1}=x_{5}, t_{2}=f\left(x_{3}, f\left(x_{4}, f\left(x_{2}, x_{7}, x_{10}\right), x_{5}\right), x_{5}\right)$ and $t_{3}=f\left(f\left(x_{5}, x_{4}, f\left(x_{2}, x_{7}, x_{10}\right)\right)\right.$, $\left.x_{1}, x_{6}\right)$. Then

$$
\begin{array}{rll}
\operatorname{seq}^{\mathrm{t}}\left(\chi_{5}^{(1)}\right)=(1) & \text { and } & \operatorname{depth}^{\mathrm{t}}\left(\chi_{5}^{(1)}\right)=1 ; \\
\operatorname{seq}^{\mathrm{t}}\left(x_{5}^{(2)}\right)=(2,2,3) & \text { and } & \operatorname{depth}^{\mathrm{t}}\left(\chi_{5}^{(2)}\right)=3 ; \\
\operatorname{seq}^{\mathrm{t}}\left(x_{5}^{(3)}\right)=(2,3) & \text { and } & \operatorname{depth}^{\mathrm{t}}\left(x_{5}^{(3)}\right)=2 ;
\end{array}
$$




$$
\begin{array}{rll}
\operatorname{seq}^{\mathrm{t}}\left(x_{5}^{(4)}\right)=(3,1,1) & \text { and } & \operatorname{depth}^{\mathrm{t}}\left(x_{5}^{(4)}\right)=3 ; \\
\operatorname{seq}^{\mathrm{t}}\left(\mathrm{f}\left(x_{2}, x_{7}, x_{10}\right)^{(1)}\right)=(2,2,2) & \text { and } & \operatorname{depth}^{\mathrm{t}}\left(\mathrm{f}\left(x_{2}, x_{7}, x_{10}\right)^{(1)}\right)=3 ; \\
\operatorname{seq}^{\mathrm{t}}\left(\mathrm{f}\left(x_{2}, x_{7}, x_{10}\right)^{(2)}\right)=(3,1,3) & \text { and } & \operatorname{depth}^{\mathrm{t}}\left(f\left(x_{2}, x_{7}, x_{10}\right)^{(2)}\right)=3 ; \\
\operatorname{seq}^{t_{3}}\left(f\left(x_{2}, x_{7}, x_{10}\right)^{(1)}\right)=(1,3) & \text { and } & \operatorname{depth}^{t_{3}}\left(f\left(x_{2}, x_{7}, x_{10}\right)^{(1)}\right)=2 ; \\
\operatorname{seq}^{\mathrm{t}}\left(x_{10}^{(1)}\right)=(2,2,2,3) & \text { and } & \operatorname{depth}^{\mathrm{t}}\left(x_{10}^{(1)}\right)=(4) ; \\
\operatorname{seq}^{\mathrm{t}}\left(x_{10}^{(2)}\right)=(3,1,3,3) & \text { and } & \operatorname{depth}^{\mathrm{t}}\left(x_{10}^{(2)}\right)=4 ; \\
\operatorname{seq}^{\mathrm{t}_{3}}\left(x_{10}^{(1)}\right)=(1,3,3) & \text { and } & \operatorname{depth}^{\mathrm{t}_{3}}\left(x_{10}^{(1)}\right)=3 .
\end{array}
$$

Let $t, s_{1}, s_{2}, \ldots, s_{k} \in W_{(n)}(X) \backslash X$ and $x_{i} \in \operatorname{var}(t)$. We donote $x_{i}^{(j)}$ - variable $x_{i}$ occurring in the $j^{\text {th }}$ order of $t$ (from the left);

$x_{i}^{\left(j, j_{1}\right)}$ - variable $x_{i}^{(j)}$ occurring in the $j_{1}^{\text {th }}$ order of $\widehat{\sigma}_{s_{1}}[t]$ (from the left);

$x_{i}^{\left(j, j_{1}, j_{2}\right)}$ - variable $x_{i}^{\left(j, j_{1}\right)}$ occurring in the $j_{2}^{\text {th }}$ order of $\widehat{\sigma}_{s_{2}}\left[\widehat{\sigma}_{s_{1}}[t]\right]$ (from the left).

Similarly,

$$
\begin{aligned}
& x_{i}^{\left(j, j_{1}, j_{2}, \ldots, j_{k}\right)}-\text { variable } x_{i}^{\left(j, j_{1}, \ldots, j_{k-1}\right)} \text { occurring in the } j_{k}^{\text {th }} \text { order of } \\
& \widehat{\sigma}_{s_{k}}\left[\widehat { \sigma } _ { s _ { k - 1 } } \left[\ldots\left[\widehat{\sigma}_{s_{2}}\left[\widehat{\sigma}_{s_{1}}[t]\right] \ldots\right]\right.\right. \text { (from the left). }
\end{aligned}
$$

Theorem 1 Let $\mathrm{t}, \mathrm{s} \in \mathrm{W}_{(\mathrm{n})}(\mathrm{X}) \backslash \mathrm{X}$ and $\mathrm{x}_{\mathrm{i}}^{(\mathrm{j})} \in \operatorname{var}(\mathrm{t})$ for some $\mathrm{i}, \mathrm{j} \in \mathbb{N}$ and let $\operatorname{seq}^{\mathrm{t}}\left(x_{i}^{(j)}\right)=i_{1}, \ldots, i_{m}$. Then $x_{i_{1}}, \ldots, x_{i_{m}} \in \operatorname{var}(s) \cap X_{n}$ if and only if $x_{i}^{\left(j, j_{1}\right)} \in$ $\operatorname{var}\left(\widehat{\sigma}_{s}[t]\right)=\operatorname{var}\left(\sigma_{s} \circ_{G} \sigma_{t}\right)$ for some $j_{1} \in \mathbb{N}$ and $\operatorname{seq}^{\widehat{\sigma}_{s}[t]}\left(x_{i}^{\left(j, j j_{1}\right)}\right)=\left(a_{i_{1}}, \ldots, a_{i_{m}}\right)$ where $\mathrm{a}_{\mathfrak{i}_{1}}$ is a sequence of natural number $\mathrm{p}_{1}, \ldots, \mathrm{p}_{\mathrm{q}}$ such that $\left(\mathrm{p}_{1}, \ldots, \mathrm{p}_{\mathrm{q}}\right)=$ $\operatorname{seq}^{\mathrm{s}}\left(\mathrm{x}_{\mathfrak{i}_{l}}^{h_{l}}\right)$ for some $\mathrm{h}_{\mathrm{l}} \in \mathbb{N}$ and for all $\mathrm{l} \in\{1, \ldots, \mathrm{m}\}$.

Proof. $(\Rightarrow)$ By Lemma 1.

$(\Leftarrow)$ Assume that $x_{i}^{\left(j, j_{1}\right)} \in \operatorname{var}\left(\widehat{\sigma}_{s}[t]\right)=\operatorname{var}\left(\sigma_{s} \circ_{G} \sigma_{t}\right)$ for some $j_{1} \in \mathbb{N}$ and $\operatorname{seq}^{\widehat{\sigma}_{s}[t]}\left(x_{i}^{\left(j, j_{1}\right)}\right)=\left(a_{i_{1}}, \ldots, a_{i_{m}}\right)$ where $a_{i_{l}}$ is a sequence of natural number $p_{1}, \ldots, p_{q}$ such that $\left(p_{1}, \ldots, p_{q}\right)=\operatorname{seq}^{s}\left(x_{i_{l}}^{h_{l}}\right)$ for some $h_{l} \in \mathbb{N}$ and for all $l \in\{1, \ldots, \mathrm{m}\}$. Then

$$
v \mathrm{~b}^{\widehat{\sigma}_{s}[t]}\left(x_{i}^{(j)}\right)=v b^{s}\left(x_{i_{1}}\right) \times v b^{s}\left(x_{i_{2}}\right) \times \ldots \times v b^{s}\left(x_{i_{m}}\right) .
$$

Suppose that $x_{i_{k}} \notin \operatorname{var}(s) \cap X_{n}$ for some $1 \leq k \leq m$, so $v b^{s}\left(x_{i_{z}}\right)=0$, i.e. $v \mathrm{~b}^{\widehat{\sigma}_{s}}[\mathrm{t}]\left(x_{i}^{(j)}\right)=0$, which contradicts to our assumption. Hence $x_{i_{1}}, \ldots, x_{i_{m}} \in$ $\operatorname{var}(s) \cap X_{n}$.

Example 4 Let $\tau=(3)$ and let $t=f\left(x_{2}, f\left(x_{4}, x_{5}, x_{2}\right), f\left(x_{2}, x_{6}, x_{7}\right)\right)$ and $s=$ $f\left(x_{3}, x_{1}, x_{3}\right)$. Then $\operatorname{seq}^{\mathrm{t}}\left(x_{2}^{(1)}\right)=(1), \operatorname{seq}^{\mathrm{t}}\left(x_{2}^{(2)}\right)=(2,3), \operatorname{seq}^{\mathrm{t}}\left(x_{2}^{(3)}\right)=(3,1)$ 
and $\operatorname{seq}^{\mathrm{t}}\left(x_{7}^{(1)}\right)=(3,3)$. By Theorem 1 , there is $x_{2}^{(1, h)}, x_{2}^{\left(3, \mathrm{k}_{1}\right)}, x_{2}^{\left(3, \mathrm{k}_{2}\right)}, x_{7}^{\left(1, \mathrm{l}_{1}\right)}$, $x_{7}^{\left(1, l_{2}\right)}, x_{7}^{\left(1, l_{3}\right)}, x_{7}^{\left(1, l_{4}\right)} \in \operatorname{var}\left(\widehat{\sigma}_{s}[t]\right)$ for some $h, k_{1}, k_{2}, l_{1}, l_{2,3}, l_{4} \in \mathbb{N}$ and

$$
\begin{aligned}
& \operatorname{seq}^{\widehat{\sigma}_{s}[t]}\left(x_{2}^{(1, h)}\right)=(2)=\operatorname{seq}^{\widehat{\sigma}_{s}[t]}\left(x_{2}^{(1,2)}\right) \text { where } \operatorname{seq}^{\mathrm{s}}\left(x_{1}^{(1)}\right)=(2) \\
& \operatorname{seq}^{\widehat{\sigma}_{s}[t]}\left(x_{2}^{\left(3, k_{1}\right)}\right)=(1,2)=\operatorname{seq}^{\widehat{\sigma}_{s}[t]}\left(x_{2}^{(3,1)}\right) \text { where } \operatorname{seq}^{s}\left(x_{3}^{(1)}\right)=(1) \text { and } \\
& \operatorname{seq}^{\mathrm{s}}\left(x_{1}^{(1)}\right)=(2) \\
& \operatorname{seq}^{\widehat{\sigma}_{s}[t]}\left(x_{2}^{\left(3, k_{2}\right)}\right)=(3,2)=\operatorname{seq}^{\widehat{\sigma}_{s}[t]}\left(x_{2}^{(3,3)}\right) \text { where } \operatorname{seq}^{s}\left(x_{3}^{(2)}\right)=(3) \text { and } \\
& \operatorname{seq}^{s}\left(x_{1}^{(1)}\right)=(2) \\
& \operatorname{seq}^{\widehat{\sigma}_{s}[t]}\left(x_{7}^{\left(1, l_{1}\right)}\right)=(1,1)=\operatorname{seq}^{\widehat{\sigma}_{s}[t]}\left(x_{7}^{(1,1)}\right) \text { where } \operatorname{seq}^{\mathrm{s}}\left(x_{3}^{(1)}\right)=(1) \text { and } \\
& \operatorname{seq}^{s}\left(x_{3}^{(1)}\right)=(1) \\
& \operatorname{seq}^{\widehat{\sigma}_{s}[t]}\left(x_{7}^{\left(1, l_{2}\right)}\right)=(1,3)=\operatorname{seq}^{\widehat{\sigma}_{s}[t]}\left(x_{7}^{(1,2)}\right) \text { where } \operatorname{seq}^{s}\left(x_{3}^{(1)}\right)=(1) \text { and } \\
& \operatorname{seq}^{\mathrm{s}}\left(x_{3}^{(2)}\right)=(3) \\
& \operatorname{seq}^{\widehat{\sigma}_{s}[t]}\left(x_{7}^{\left(1, l_{3}\right)}\right)=(3,1)=\operatorname{seq}^{\widehat{\sigma}_{s}[t]}\left(x_{7}^{(1,3)}\right) \text { where } \operatorname{seq}^{\mathrm{s}}\left(x_{3}^{(2)}\right)=(3) \text { and } \\
& \operatorname{seq}^{s}\left(x_{3}^{(1)}\right)=(1) \\
& \operatorname{seq}^{\widehat{\sigma}_{s}[t]}\left(x_{7}^{\left(1, l_{4}\right)}\right)=(3,3)=\operatorname{seq}^{\widehat{\sigma}_{s}[t]}\left(x_{7}^{(1,4)}\right) \text { where } \operatorname{seq}^{s}\left(x_{3}^{(2)}\right)=(3) \text { and } \\
& \operatorname{seq}^{\mathrm{s}}\left(x_{3}^{(2)}\right)=(3) \text {. }
\end{aligned}
$$

Since $x_{2} \notin \operatorname{var}(s)$, so $x_{2}^{(2, i)} \notin \operatorname{var}\left(\widehat{\sigma}_{s}[t]\right)$ for all $i \in \mathbb{N}$. Consider,

$$
\begin{aligned}
\widehat{\sigma}_{s}[t] & =\widehat{\sigma}_{s}\left[f\left(x_{2}^{(1)}, f\left(x_{4}, x_{5}, x_{2}^{(2)}\right), f\left(x_{2}^{(3)}, x_{6}, x_{7}^{(1)}\right)\right)\right] \\
& =S^{3}\left(f\left(x_{3}, x_{1}, x_{3}\right), \widehat{\sigma}_{s}\left[x_{2}^{(1)}\right], \widehat{\sigma}_{s}\left[f\left(x_{4}, x_{5}, x_{2}^{(2)}\right)\right], \widehat{\sigma}_{s}\left[f\left(x_{2}^{(3)}, x_{6}, x_{7}^{(1)}\right)\right]\right) \\
& =f\left(f\left(x_{7}^{(1,1)}, x_{2}^{(3,1)}, x_{7}^{(1,2)}\right), x_{2}^{(1,2)}, f\left(x_{7}^{(1,3)}, x_{2}^{(3,3)}, x_{7}^{(1,4)}\right)\right) \\
& =f\left(f\left(x_{7}, x_{2}, x_{7}\right), x_{2}, f\left(x_{7}, x_{2}, x_{7}\right)\right) .
\end{aligned}
$$

Corollary 1 Let $\mathrm{t}, \mathrm{s} \in \mathrm{W}_{(\mathrm{n})}(\mathrm{X}) \backslash \mathrm{X}$ and $\chi_{i}^{(\mathrm{j})} \in \operatorname{var}(\mathrm{t})$ for some $\mathrm{i}, \mathrm{j} \in \mathbb{N}$ such that $\operatorname{seq}^{t}\left(x_{i}^{(j)}\right)=\left(i_{1}, i_{2}, \ldots, i_{m}\right)$ for some $i_{1}, i_{2}, \ldots, i_{m} \in\{1, \ldots, n\}$ and $x_{i_{k}} \in \operatorname{var}(s)$ for all $1 \leq \mathrm{k} \leq \mathrm{m}$. Then there is $j_{1} \in \mathbb{N}$ such that

$$
\operatorname{depth}^{\widehat{\sigma}_{s}[t]}\left(x_{i}^{\left(j, j_{1}\right)}\right)=\operatorname{depth}^{s}\left(x_{i_{1}}^{\left(l_{1}\right)}\right)+\operatorname{depth}^{s}\left(x_{i_{2}}^{\left(l_{2}\right)}\right)+\ldots+\operatorname{depth}^{s}\left(x_{i_{m}}^{\left(l_{m}\right)}\right)
$$

for some $l_{1}, l_{2}, \ldots, l_{m} \in \mathbb{N}$, and

$$
v b^{\widehat{\sigma}_{s}[t]}\left(x_{i}^{(j)}\right)=v b^{s}\left(x_{i_{1}}\right) \times v b^{s}\left(x_{i_{2}}\right) \times \ldots \times v b^{s}\left(x_{i_{m}}\right) .
$$

Let $v \mathrm{~b}^{\mathrm{t}}\left(\mathrm{x}_{\mathrm{i}}\right)=\mathrm{d}$.

$$
\text { If } x_{i} \in X_{n} \text {, then } v \mathrm{~b}^{\widehat{\sigma}_{s}[t]}\left(x_{i}\right)=\sum_{j=1}^{d} v b^{\widehat{\sigma}_{s}[t]}\left(x_{i}^{(j)}\right) \text {. }
$$


If $x_{i} \in X \backslash X_{n}$ where $x_{i} \notin \operatorname{var}(s)$, then $v \mathrm{~b}^{\widehat{\sigma}_{s}[t]}\left(x_{i}\right)=\sum_{j=1}^{d} v b^{\widehat{\sigma}_{s}[t]}\left(x_{i}^{(j)}\right)$.

\section{Main results}

In this section, we will show that the set of all completely regular elements and the set of all intra-regular elements in $\mathrm{Hyp}_{\mathrm{G}}(2)$ are the same. First, we recall definitions of regular and completely regular elements and then we characterize all completely regular elements in $\mathrm{Hyp}_{\mathrm{G}}(2)$.

Definition 3 [6] An element a of a semigroup $S$ is called regular if there exists $x \in S$ such that $a x a=a$.

Definition 4 [9] An element a of a semigroup $S$ is called completely regular if there exists $b \in S$ such that $a=a b a$ and $a b=b a$.

Let $\sigma_{t} \in \operatorname{Hyp}_{\mathrm{G}}(2)$. We denote

$R_{1}:=\left\{\sigma_{x_{i}} \mid x_{i} \in X\right\}$

$R_{2}:=\left\{\sigma_{t} \mid \operatorname{var}(t) \cap X_{2}=\emptyset\right\} ;$

$R_{3}:=\left\{\sigma_{t} \mid t=f\left(t_{1}, t_{2}\right)\right.$ where $t_{i}=x_{j}$ for some $i, j \in\{1,2\}$ and $\operatorname{var}(t) \cap X_{2}=$ $\left.\left\{x_{j}\right\}\right\} \cup\left\{\sigma_{f\left(x_{1}, x_{2}\right)}, \sigma_{f\left(x_{2}, x_{1}\right)}\right\}$

$\mathrm{CR}\left(\mathrm{R}_{3}\right):=\left\{\sigma_{t} \mid t=f\left(t_{1}, t_{2}\right)\right.$ where $t_{i}=x_{i}$ for some $i \in\{1,2\}$ and $\operatorname{var}(t) \cap$ $\left.X_{2}=\left\{x_{i}\right\}\right\} \cup\left\{\sigma_{f\left(x_{1}, x_{2}\right)}, \sigma_{f}\left(x_{2}, x_{1}\right)\right\}$.

It was shown in [10] and [3] that $\bigcup_{i=1}^{3} R_{i}$ is the set of all regular elements in $\operatorname{Hyp}_{\mathrm{G}}(2)$ and $\mathrm{CR}\left(\mathrm{Hyp}_{\mathrm{G}}(2)\right):=\mathrm{CR}\left(\mathrm{R}_{3}\right) \cup \mathrm{R}_{1} \cup \mathrm{R}_{2}$ is the set of all completely regular elements in $\operatorname{Hyp}_{\mathrm{G}}(2)$, respectively.

Definition 5 [9] An element a of a semigroup $S$ is called intra-regular if there is $b \in S$ such that $a=b a a b$.

Theorem 2 [3] Let $\mathrm{S}$ be a semigroup and $\mathrm{a} \in \mathrm{S}$. If $\mathrm{a}$ is completely regular, then a is intra-regular.

Corollary 2 [3] Let $\sigma_{\mathrm{t}} \in \mathrm{CR}\left(\mathrm{Hyp}_{\mathrm{G}}(2)\right)$. Then $\sigma_{\mathrm{t}}$ is intra-regular in $\mathrm{Hyp} \mathrm{g}_{\mathrm{G}}(2)$.

Lemma 2 Let $\mathrm{t}=\mathrm{f}\left(\mathrm{t}_{1}, \mathrm{x}_{1}\right)$ where $\mathrm{t}_{1} \in \mathrm{W}_{(2)}(\mathrm{X}) \backslash \mathrm{X}_{2}$. Then $\sigma_{\mathrm{t}}$ is not intraregular in $\mathrm{Hyp}_{\mathrm{G}}(2)$. 
Proof. Let $t=f\left(t_{1}, x_{1}\right)$ where $t_{1} \in W_{(2)}(X) \backslash X_{2}$. For each $u \in X$, we get $\sigma_{\mathfrak{u}} \circ_{\mathrm{G}} \sigma_{\mathrm{t}}^{2} \circ_{\mathrm{G}} \sigma_{v} \neq \sigma_{\mathrm{t}}$ and $\sigma_{v} \circ_{\mathrm{G}} \sigma_{\mathrm{t}}^{2} \circ_{\mathrm{G}} \sigma_{\mathfrak{u}} \neq \sigma_{\mathrm{t}}$ for all $v \in \mathrm{W}_{(2)}(\mathrm{X})$. Let $u, v \in$ $W_{(2)}(X) \backslash X$ where $u=f\left(u_{1}, u_{2}\right)$ and $v=f\left(v_{1}, v_{2}\right)$ for some $u_{1}, u_{2}, v_{1}, v_{2} \in$ $W_{(2)}(X)$, we will show that $\sigma_{\mathfrak{u}} \circ_{\mathrm{G}} \sigma_{\mathrm{t}}^{2} \circ_{\mathrm{G}} \sigma_{v} \neq \sigma_{\mathrm{t}}$. If $\mathrm{t}_{1} \in X \backslash X_{2}$ then $\mathrm{x}_{2} \notin$ $\operatorname{var}(\mathrm{t})$. By Theorem 1, $\mathrm{x}_{1} \notin \operatorname{var}\left(\widehat{\sigma}_{\mathrm{t}}[\mathrm{t}]\right)=\operatorname{var}\left(\sigma_{\mathrm{t}}^{2}\right)$, i.e. $\operatorname{var}\left(\sigma_{\mathrm{t}}^{2}\right) \cap \mathrm{X}_{2}=\emptyset$. Hence $\sigma_{\mathfrak{u}} \circ_{\mathrm{G}} \sigma_{\mathrm{t}}^{2} \circ_{\mathrm{G}} \sigma_{v} \neq \sigma_{\mathrm{t}}$. If $\mathrm{t}_{1} \in \mathrm{W}_{(2)}(\mathrm{X}) \backslash \mathrm{X}$,

$$
\sigma_{\mathrm{t}}^{2}(f)=\widehat{\sigma}_{t}[t]=S^{2}\left(f\left(t_{1}, x_{1}\right), \widehat{\sigma}_{t}\left[t_{1}\right], x_{1}\right)=f\left(w_{1}, w_{2}\right)
$$

where $w_{1}=S^{2}\left(t_{1}, \widehat{\sigma}_{t}\left[t_{1}\right], x_{1}\right)$ and $w_{2}=S^{2}\left(x_{1}, \widehat{\sigma}_{t}\left[t_{1}\right], x_{1}\right)=\widehat{\sigma}_{t}\left[t_{1}\right]$. Let $w=$ $\mathrm{f}\left(w_{1}, w_{2}\right)$. Since $\mathrm{t}_{1} \notin X$, so $w_{1} \notin X$ and $w_{2}=\widehat{\sigma}_{t}\left[\mathrm{t}_{1}\right] \notin X$. Consider

$$
\sigma_{\mathrm{t}}^{2} \circ_{\mathrm{G}} \sigma_{v}(f)=\widehat{\sigma}_{w}[v]=S^{2}\left(f\left(w_{1}, w_{2}\right), \widehat{\sigma}_{w}\left[v_{1}\right], \widehat{\sigma}_{w}\left[v_{2}\right]\right)=f\left(s_{1}, s_{2}\right)
$$

where $s_{i}=S^{2}\left(w_{i}, \widehat{\sigma}_{w}\left[v_{1}\right], \widehat{\sigma}_{w}\left[v_{2}\right]\right)$ for all $i \in\{1,2\}$. Since $w_{i} \notin X$ for all $i \in\{1,2\}$, $s_{i} \notin X$ for all $i \in\{1,2\}$. Then $\widehat{\sigma}_{u}\left[s_{i}\right] \notin X$ for all $i \in\{1,2\}$. Consider

$$
\sigma_{\mathfrak{u}} \circ_{G} \sigma_{\mathfrak{t}}^{2} \circ_{G} \sigma_{v}(f)=S^{2}\left(f\left(u_{1}, u_{2}\right), \widehat{\sigma}_{\mathfrak{u}}\left[s_{1}\right], \widehat{\sigma}_{\mathfrak{u}}\left[s_{2}\right]\right)=f\left(r_{1}, r_{2}\right)
$$

where $r_{i}=S^{2}\left(u_{i}, \widehat{\sigma}_{u}\left[s_{1}\right], \widehat{\sigma}_{u}\left[s_{2}\right]\right)$ for all $i \in\{1,2\}$. If $u_{2} \in W_{(2)}(X) \backslash X$ or $u_{2} \in X_{2}$ then $r_{2} \notin X$. If $u_{2} \in X \backslash X_{2}$ then $u_{2}=r_{2}$. So $r_{2} \neq x_{1}$. Therefore $\sigma_{\mathfrak{u}} \circ_{G} \sigma_{t}^{2} \circ_{G} \sigma_{v} \neq$ $\sigma_{t}$. Hence $\sigma_{t}$ is not intra-regular in $\operatorname{Hyp}_{\mathrm{G}}(2)$.

Lemma 3 Let $\mathrm{t}=\mathrm{f}\left(\mathrm{x}_{2}, \mathrm{t}_{2}\right)$ where $\mathrm{t}_{2} \in \mathrm{W}_{(2)}(\mathrm{X}) \backslash \mathrm{X}_{2}$. Then $\sigma_{\mathrm{t}}$ is not intraregular in $\mathrm{Hyp}_{\mathrm{G}}(2)$.

Proof. The proof is similar to the proof of Lemma 2.

Lemma 4 Let $\mathrm{t}=\mathrm{f}\left(\mathrm{x}_{1}, \mathrm{t}_{2}\right)$ where $\mathrm{t}_{2} \in \mathrm{W}_{(2)}(\mathrm{X}) \backslash \mathrm{X}_{2}$ and $\mathrm{x}_{2} \in \operatorname{var}(\mathrm{t})$. Then $\sigma_{\mathrm{t}}$ is not intra-regular in $\mathrm{Hyp}_{\mathrm{G}}(2)$.

Proof. Assume that $t=f\left(x_{1}, t_{2}\right)$ where $t_{2} \in W_{(2)}(X) \backslash X_{2}$ and $x_{2} \in \operatorname{var}(t)$. Let $\mathrm{m}=\max \left\{\operatorname{depth}^{\mathrm{t}}\left(x_{2}^{(\mathfrak{i})}\right) \mid x_{2}^{(i)} \in \operatorname{var}(\mathrm{t})\right.$ for some $\left.i \in \mathbb{N}\right\}(*)$, then there exists $\mathrm{h} \in \mathbb{N}$ such that $\operatorname{seq}^{\mathrm{t}}\left(x_{2}^{(\mathrm{h})}\right)=\left(\mathfrak{i}_{1}, \mathfrak{i}_{2}, \ldots, \mathfrak{i}_{\mathrm{m}}\right)$ where $i_{1}, \mathfrak{i}_{2}, \ldots, i_{\mathrm{m}} \in\{1,2\}$. It means $x_{2}^{(h)}=\pi_{i_{m}} \circ \pi_{i_{m-1}} \circ \ldots \circ \pi_{i_{1}}(t)$ where maps $\pi_{i_{1}}, \ldots, \pi_{i_{m-1}}, \pi_{i_{m}}$ are defined on $W_{(2)}(X) \backslash X_{2}$ to $W_{(2)}(X)$. Since $x_{2}^{(h)} \in \operatorname{var}\left(t_{2}\right), \pi_{i_{1}}(t)=t_{2}$, i.e. $i_{1}=2$. So $\operatorname{seq}^{\mathrm{t}}\left(x_{2}^{(\mathrm{h})}\right)=\left(2, i_{2}, \ldots, i_{\mathrm{m}}\right)$. By Theorem 1 , there is $x_{2}^{\left(\mathrm{h}, \mathrm{h}_{1}\right)} \in \operatorname{var}\left(\widehat{\sigma}_{\mathrm{t}}[\mathrm{t}]\right)=$ $\operatorname{var}\left(\sigma_{t}^{2}\right)$ for some $h_{1} \in \mathbb{N}$ such that

$$
\operatorname{seq}^{\sigma_{\mathfrak{t}}^{2}}\left(x_{2}^{\left(h, h_{1}\right)}\right)=\left(2, i_{2}, \ldots, i_{m}, a_{i_{2}}, \ldots, a_{i_{m}}\right)
$$


where $\left(2, i_{2}, \ldots, i_{m}\right)=\operatorname{seq}^{t}\left(x_{2}^{(h)}\right)$ and $a_{i_{z}}$ is a sequence of natural numbers such that $\left(a_{i_{z}}\right)=\operatorname{seq}^{s}\left(x_{i_{z}}^{\left(h_{i_{z}}\right)}\right)$ for some $h_{i_{z}} \in \mathbb{N}$ and for all $2 \leq z \leq m$. [Note: $x_{2}^{(h)}$ is a variable $x_{2}$ occurring in the $h^{\text {th }}$ order of $t$ (from the left) and $x_{2}^{\left(h, h_{1}\right)}$ is a variable $x_{2}^{(h)}$ occurring in the $h_{1}^{\text {th }}$ order of $\sigma_{t}^{2}$ (from the left)]. Instead of a sequence $a_{i_{2}}, \ldots, a_{i_{m}}$, we write a sequence of natural numbers $w_{1}, \ldots, w_{d}$ for some $\mathrm{d} \in \mathbb{N}$ and $w_{1}, \ldots, w_{\mathrm{d}} \in\{1,2\}$. Then

$$
\operatorname{seq}^{\sigma_{t}^{2}}\left(x_{2}^{\left(h, h_{1}\right)}\right)=\left(2, i_{2}, \ldots, i_{m}, w_{1}, \ldots, w_{d}\right) .
$$

Suppose that there exist $u, v \in W_{(2)}(X)$ such that $\sigma_{\mathfrak{u}} \circ_{G} \sigma_{t}^{2} \circ_{G} \sigma_{v}=\sigma_{t}(* *)$, i.e. $u=f\left(x_{1}, u_{2}\right)$ and $v=f\left(x_{1}, v_{2}\right)$ for some $u_{2}, v_{2} \in W_{2}(X)$ where $x_{2} \in \operatorname{var}\left(u_{2}\right) \cap$ $\operatorname{var}\left(v_{2}\right)$. Choose $x_{2}^{(j)} \in \operatorname{var}(v)$ for some $j \in \mathbb{N}$. Then $\operatorname{seq}^{v}\left(x_{2}^{(j)}\right)=\left(2, p_{1}, \ldots, p_{q}\right)$ for some $p_{1}, \ldots, p_{q} \in\{1,2\}$ and for some $q \in \mathbb{N}$. By Theorem 1 , there is $x_{2}^{\left(j, j_{1}\right)} \in \operatorname{var}\left(\sigma_{\mathrm{t}}^{2} \circ_{\mathrm{G}} \sigma_{v}\right)$ for some $j_{1} \in \mathbb{N}$ such that

$$
\operatorname{seq}^{\sigma_{\mathfrak{t}}^{2} \circ_{G} \sigma_{v}}\left(x_{2}^{\left(j, j_{1}\right)}\right)=\left(2, i_{2}, \ldots, i_{m}, w_{1}, \ldots, w_{d}, a_{p_{1}}, \ldots, a_{p_{q}}\right)
$$

where $\left(2, i_{2}, \ldots, i_{m}, w_{1}, \ldots, w_{d}\right)=\operatorname{seq}^{\sigma_{\mathfrak{t}}^{2}}\left(x_{2}^{\left(h, h_{1}\right)}\right)$ and $a_{p_{z}}$ is a sequence of natural numbers such that $\left(a_{\mathfrak{p}_{z}}\right)=\operatorname{seq}^{\mathrm{s}}\left(x_{\mathfrak{p}_{z}}^{\left(\mathrm{l}_{z}\right)}\right)$ for some $\mathrm{l}_{z} \in \mathbb{N}$ and for all $1 \leq z \leq \mathrm{q}$. [Note: $x_{2}^{(j)}$ is a variable $x_{2}$ occurring in the $j^{\text {th }}$ order of $v$ (from the left) and $x_{2}^{\left(j, j_{1}\right)}$ is a variable $x_{2}^{(j)}$ occurring in the $j_{1}^{\text {th }}$ order of $\sigma_{t}^{2} \circ_{G} \sigma_{v}$ (from the left)]. Instead of a sequence $a_{p_{1}}, \ldots, a_{p_{q}}$ we write a sequence of natural numbers $w_{\mathrm{d}+1}, \ldots, w_{\mathrm{k}}$ for some $k \in \mathbb{N}$ and $w_{\mathrm{d}+1}, \ldots, w_{\mathrm{k}} \in\{1,2\}$. Then

$$
\operatorname{seq}^{\sigma_{\mathrm{t}}^{2} \circ_{\mathrm{G}} \sigma_{v}}\left(x_{2}^{\left(j, j_{1}\right)}\right)=\left(2, i_{2}, \ldots, i_{\mathrm{m}}, w_{1}, \ldots, w_{\mathrm{d}}, w_{\mathrm{d}+1}, \ldots, w_{\mathrm{k}}\right) \text {. }
$$

By Theorem 1, we have $x_{2}^{\left(j, j, j, j_{2}\right)} \in \operatorname{var}\left(\sigma_{u} \circ_{G} \sigma_{t}^{2} \circ_{G} \sigma_{v}\right)$ for some $j_{2} \in \mathbb{N}$. By Corollary 1, we have

$$
\begin{aligned}
& \operatorname{depth}^{\sigma_{\mathfrak{u}} \circ_{G} \sigma_{\mathfrak{t}}^{2} \circ_{G} \sigma_{v}}\left(x_{2}^{\left(j, j_{1}, j_{2}\right)}\right)=\operatorname{depth}^{\mathfrak{u}}\left(x_{2}^{\left(b_{1}\right)}\right)+\operatorname{depth}^{\mathfrak{u}}\left(x_{\mathfrak{i}_{2}}^{\left(b_{2}\right)}\right)+\ldots+\operatorname{depth}^{\mathfrak{u}}\left(x_{\mathfrak{i}_{\mathfrak{m}}}^{\left(\mathrm{b}_{\mathfrak{m}}\right)}\right) \\
& +\operatorname{depth}^{\mathfrak{u}}\left(x_{w_{1}}^{\left(b_{\mathfrak{m}+1}\right)}\right)+\ldots+\operatorname{depth}^{\mathfrak{u}}\left(x_{w_{d}}^{\left(b_{\mathfrak{d}+\mathrm{d}}\right)}\right) \\
& +\operatorname{depth}^{\mathfrak{u}}\left(x_{w_{d+1}}^{\left(b_{\mathfrak{m}+\mathrm{d}+1}\right)}\right)+\ldots+\operatorname{depth}^{\mathfrak{u}}\left(x_{w_{\mathfrak{k}}}^{\left(\mathrm{b}_{\mathfrak{m}+\mathrm{k}}\right)}\right) \\
& >\mathrm{m}
\end{aligned}
$$

for some $b_{1}, \ldots, b_{m}, b_{m+1}, \ldots, b_{m+d}, b_{m+d+1}, \ldots, b_{m+k} \in \mathbb{N}$, which contradicts to $(*)$ and $(* *)$. Therefore $\sigma_{t}$ is not intra-regular in $\operatorname{Hyp}_{\mathrm{G}}(2)$.

Lemma 5 Let $\mathrm{t}=\mathrm{f}\left(\mathrm{t}_{1}, \mathrm{x}_{2}\right)$ where $\mathrm{t}_{1} \in \mathrm{W}_{(2)}(\mathrm{X}) \backslash \mathrm{X}_{2}$ and $\mathrm{x}_{1} \in \operatorname{var}(\mathrm{t})$. Then $\sigma_{\mathrm{t}}$ is not intra-regular in $\mathrm{Hyp}_{\mathrm{G}}(2)$. 
Proof. The proof is similar to the proof of Lemma 4.

Lemma 6 If $\mathrm{t}=\mathrm{f}\left(\mathrm{t}_{1}, \mathrm{t}_{2}\right)$ where $\mathrm{t}_{1}, \mathrm{t}_{2} \in \mathrm{W}_{(2)}(\mathrm{X}) \backslash \mathrm{X}_{2}$ and $\operatorname{var}(\mathrm{t}) \cap \mathrm{X}_{2} \neq \emptyset$ then $\sigma_{\mathrm{t}}$ is not intra-regular in $\mathrm{Hyp}_{\mathrm{G}}(2)$.

Proof. Let $t=f\left(t_{1}, t_{2}\right)$ where $t_{1}, t_{2} \in W_{(2)}(X) \backslash X_{2}$ and $\operatorname{var}(t) \cap X_{2} \neq \emptyset$.

Case1: $\operatorname{var}(t) \cap X_{2}=\left\{x_{i}\right\}$ for some $i \in\{1,2\}$. Let $j \in\{1,2\}$ where $i \neq j$.

If $j$ is occurring in $\operatorname{seq}^{t}\left(x_{i}^{(h)}\right)$ for all $x_{i}^{(h)} \in \operatorname{var}(t)$ then $\operatorname{var}\left(\sigma_{t}^{2}\right) \cap X_{2}=\emptyset$, i.e. $\sigma_{\mathfrak{u}} \circ_{\mathrm{G}} \sigma_{\mathrm{t}}^{2} \circ_{\mathrm{G}} \sigma_{v} \neq \sigma_{\mathrm{t}}$ for all $u, v \in \mathrm{W}_{(2)}(\mathrm{X})$.

If $j$ is not occurring in $\operatorname{seq}^{t}\left(x_{i}^{(h)}\right)$ for some $x_{i}^{(h)} \in \operatorname{var}(t)$ then $\operatorname{seq}^{t}\left(x_{i}^{(h)}\right)=$ $\left(i_{1}, i_{2}, \ldots, i_{m}\right)$ where $i_{1}, i_{2}, \ldots, i_{m} \in\{i\}$ for some $m \in \mathbb{N}$. We can prove similar to the proof of Lemma 4 , then $\sigma_{u} \circ_{G} \sigma_{t}^{2} \circ_{G} \sigma_{v} \neq \sigma_{t}$ for all $u, v \in W_{(2)}(X)$.

Case2: $\operatorname{var}(\mathrm{t}) \cap \mathrm{X}_{2}=\mathrm{X}_{2}$. We can prove similar to the proof of Lemma 4, then $\sigma_{u} \circ_{G} \sigma_{t}^{2} \circ_{G} \sigma_{v} \neq \sigma_{t}$ for all $u, v \in W_{(2)}(X)$.

Therefore $\sigma_{t}$ is not intra-regular in $\operatorname{Hyp}_{G}(2)$.

Theorem $3 \mathrm{CR}\left(\mathrm{Hyp}_{\mathrm{G}}(2)\right)$ is the set of all intra-regular elements in $\mathrm{Hyp}_{\mathrm{G}}(2)$.

Proof. By Corollary 2 and by Lemma 2 to 6 .

\section{Acknowledgements}

This research was supported by Chiang Mai University, Chiang Mai 50200, Thailand.

\section{References}

[1] J. Aczèl, Proof of a Theorem of Distributive Type Hyperidentities, Algebra Universalis, 1 (1971), 1-6.

[2] V.D. Belousov, System of Quasigroups with Generalized Identities, Uspechi Mat. Nauk., 20 (1965), 75-146.

[3] A. Boonmee, S. Leeratanavalee, All Completely Regular Elements in Hyp $_{\mathrm{G}}(\mathrm{n})$, Discussiones Mathematicae General Algebra and Applications, 33 (2013), 211-219.

[4] A. Boonmee, S. Leeratanavalee, Factorisable Monoid of Generalized Hypersubstitutions of Type $\tau=(\mathrm{n})$, Acta Mathematica Universitatis Comenianae, 85 (1) (2016), 1-7. 
[5] K. Denecke, D. Lau, R. Pöschel, D. Schweigert, Hyperidentities, Hyperequational Classes and Clone Congruences, Contribution to General Algebra 7, Verlag Hölder-Pichler-Temsky, Wein, (1991), 97-118.

[6] J.M. Howie, Fundamentals of Semigroup Theory, Academic Press, London, (1995).

[7] S. Leeratanavalee, K. Denecke, Generalized Hypersubstitutions and Strongly Solid Varieties, General Algebra and Applications, Proc. of the "59th Workshop on General Algebra", "15th Conference for Young Algebraists Potsdam 2000", Shaker Verlag, (2000), 135-145

[8] W. D. Neumann, Mal'cev Conditions, Spectra and Kronecker Product, J. Austral. Math. Soc.(A), 25 (1987), 103-117.

[9] M. Petrich, N. R. Reilly, Completely Regular Semigroups, John Wiley and Sons,Inc., New York, (1999).

[10] W. Puninagool, S. Leeratanavalee, The Monoid of Generalized Hypersubstitutions of type $\tau=(\mathrm{n})$, Discussiones Mathematicae General Algebra and Applications, 30 (2010), 173-191.

[11] Sl. Shtrakov, Essential Variables and Positions in Terms, Algebra Universalis, 61 (3-4) (2009), 381-397.

[12] Sl. Shtrakov, J. Koppitz, Stable Varieties of Semigroups and Groupoids, Algebra Universalis, 75 (1) (2016), 85-106.

[13] W. Taylor, Hyperidentities and Hypervarieties, Aequationes Math., 23 (1981), 111-127. 